\title{
ON ADDITIVE REPRESENTATION FUNCTIONS
}

\author{
R. BALASUBRAMANIAN AND SUMIT GIRI
}

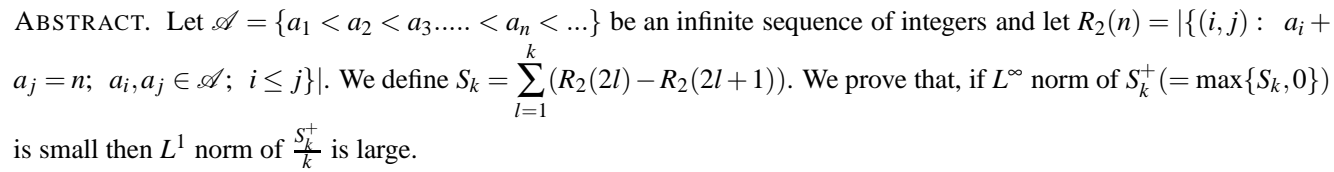

\section{INTRODUCTION}

Let $\mathscr{A}=\left\{a_{1}, a_{2}, \ldots \ldots . .,\right\}\left(0 \leq a_{1} \leq a_{2} \leq \ldots ..\right)$ be an infinite sequence of non-negative integers. Let $n \in \mathbb{N}_{0}$, denote the number of solutions of $a_{i}+a_{j}=n$, and $a_{i}+a_{j}=n(i \leq j)$ by $R_{1}(n)$ and $R_{2}(n)$, respectively. More precisely:

$$
\begin{aligned}
R_{1}(n)=R_{1}(\mathscr{A}, n) & =\sum_{a_{i}+a_{j}=n} 1 ; \\
R_{2}(n)=R_{2}(\mathscr{A}, n) & =\sum_{a_{i}+a_{j}=n} 1 ; \\
i \leq j & \\
\text { Also define, } \quad R_{3}(n)=R_{3}(\mathscr{A}, n) & =\sum_{\substack{a_{i}+a_{j}=n \\
i<j}} 1 .
\end{aligned}
$$

It is easy to check that if $\mathscr{A}$ is a full set or a complement of a finite set inside the set of natural numbers then all $R_{1}, R_{2}, R_{3}$ are monotonically increasing. Here we are interested in inverse problems. In other words how the monotonicity of one of the representation function affects the cardinality of the set $\mathscr{A}$. Erdós, Sárközy and Sós [8, 9] and the first author [2] studied the monotonicity properties of the functions $R_{1}, R_{2}, R_{3}$. It turns out that monotonicity of these three functions differs significantly.

Erdős, Sárközy and Sós [8] proved that $R_{1}(n)$ can be monotonically increasing from a certain point only in a trivial way:

Theorem A. If $R_{1}(n+1) \geq R_{1}(n)$ for all large $n$, then $\mathbb{N} \backslash \mathscr{A}$ is a finite set.

The analogous conclusion is not true in case of $R_{2}$. If we define $\mathscr{A}(N)=|\mathscr{A} \cap[1, N]|$, then first author [2] proved that,

Theorem B. If $R_{2}(n+1) \geq R_{2}(n)$ for all large $n$, then $\mathscr{A}(N)=N+O(\log N)$ [2]:

That is to say, the complement set of $\mathscr{A}$ is of order $O(\log N)$ at most. The following result was also proved in

Theorem C. If $\mathscr{A}(N)=o\left(\frac{N}{\log N}\right)$, then the function $R_{3}(n)$ can not be eventually increasing.

Again Erdos, Sarkozy and Sos [8] proved another result related to $R_{2}$ :

Theorem D. If $\lim _{n \rightarrow \infty} \frac{n-A(n)}{n}=+\infty$, then we have,

$$
\limsup _{n \rightarrow \infty} \sum_{k=1}^{\infty}\left(R_{2}(2 k)-R_{2}(2 k+1)\right)=\infty
$$

The result is tight, in fact they gave an example of a sequence $\mathscr{A}$ where $n-\mathscr{A}(n)>c \log n$ (for large $n$ and fixed constatnt $c$.) and $\limsup _{N \rightarrow \infty} \sum_{k=1}^{N}\left(R_{2}(2 k)-R_{2}(2 k+1)\right)<+\infty$.

In [2], Tang and Chen gave a quantative version of Theorem $D$, To state the theorem, let us define a few notations:

$$
\begin{aligned}
S(n) & =\sum_{k \leq n}\left(R_{2}(2 k)-R_{2}(2 k+1)\right) \\
m(N) & =N(\log N+\log \log N) .
\end{aligned}
$$


Then $L^{\infty}$ norm of $S(n)$ is defned by

$$
T(N)=\max _{n \leq N} S(n)=\max _{n \leq N} \sum_{k \leq n}\left(R_{2}(2 k)-R_{2}(2 k+1)\right) .
$$

They proved that, when the ratio $\frac{T(N)}{\mathscr{A}(N)}$ is bounded above by a small enough fixed constatnt, then $T(N) \operatorname{and} \frac{N-A(N)}{\log N}$ satisfies a simple inequality. More precisely:

Theorem E. If

$$
\begin{aligned}
T(N) & <\frac{1}{36} \mathscr{A}(N), \quad \text { then, } \\
T(N) & >\frac{1}{80 e} \frac{N-\mathscr{A}(N)}{\log N}-\frac{11}{4}-\frac{N_{1}}{8}
\end{aligned}
$$

Where $N_{1}$ is a fixed positive integer, depending only on $\mathscr{A}$

It is easy to see that under the condition (1), Theorem E implies Theorem D Now Set

$$
S^{+}(n)=\max \{S(n), 0\}
$$

and

$$
T^{+}(N)=\max _{n \leq N}\left\{S^{+}(n)\right\}
$$

Note: $T(N)$ and $T^{+}(N)$ are same unless all $\{S(n)\}_{n \leq N}$ are negative.

In this paper we again assume that $\frac{T(N)}{\mathscr{A}(N)}$ is bounded above and prove an improved inequality where we replace $L^{\infty}$ norm of $S(n)$ by $L^{1}$ norm of $\frac{S^{+}(n)}{n}$. More precisely:

Theorem 1. Let $\mathscr{A}$ be an infinite sequence of positive integers and there exists $N_{0}$ such that $T(N)<\frac{1}{36} \mathscr{A}(N)$ for all $N \geq N_{0}$. Then there exists a constatnt $N_{1}>0$ such that

$$
\sum_{n=1}^{m(N)} \frac{S_{n}^{+}}{n}>\frac{1}{10 e}(N-\mathscr{A}(N))-\frac{1}{7} \log N-c_{1} .
$$

for some constatnt $c_{1}$ depending on the first few elements of $\mathscr{A}$.

Corollary 1. If (1) holds then $T^{+}(N)>\frac{1}{17 e} \frac{N-\mathscr{A}(N)}{\log N}-\frac{1}{7}$

So if at least one of $S(n)$ is non-negetive, then $T^{+}(N)$ indeed equals to $T(N)$. In that case Corollary 1 gives Theorem E with a better constant. Corollary 1 also implies the following corollaries:

Corollary 2. If $\limsup _{N \rightarrow \infty} \frac{N-\mathscr{A}(N)}{\log N}=+\infty$, then

$$
T(N) \geq \min \left\{\frac{1}{36} \mathscr{A}(N), \frac{1}{11 e} \frac{N-\mathscr{A}(N)}{\log N}\right\}
$$

for all large $N$.

Corollary 3. If $\limsup _{N \rightarrow \infty} \frac{N-\mathscr{A}(N)}{\log N}=+\infty$, then $\limsup _{n \rightarrow \infty} S_{n}=+\infty$.

Remark 1. In the proof, we assume that $N_{1}$, mentioned in Theorem 1 is suffuciently large. But as the reader can notice, one can take any $N_{1}$, which satisfies the following:

Define $\tilde{N}$ by: $\mathscr{A}(\tilde{N})>40$,

then $N_{1}$ can be the smallest integer $N$ satisfying

$$
(0.98)^{N}<\left(\frac{40}{e \tilde{N}}\right)^{\tilde{N}} .
$$




\section{Notations and prelimanary lemmas:}

Set

$$
f(z)=\sum_{a \in \mathscr{A}} z^{a} ; \text { for }|z|<1
$$

Then

$$
f(z)^{2}=\sum_{n=1}^{\infty} R(n) z^{n}
$$

Let $\chi_{\mathscr{A}}$ be the characteristic function of $\mathscr{A}$. i.e.

$$
\chi(n)=\left\{\begin{array}{l}
1, \text { if } n \in \mathscr{A} \\
0 \text { else }
\end{array}\right.
$$

For a positive real number $Y$, define

$$
\psi(Y)=f\left(e^{-\frac{1}{Y}}\right)=\sum_{a \in \mathscr{A}} e^{-\frac{a}{Y}}
$$

and

$$
g(N)=1+4\left(1-e^{-\frac{2}{Y}}\right) \sum_{k=1}^{\infty} S_{k} e^{-\frac{2 k}{Y}} .
$$

Before proving the theorem we shall prove a few lemmas which we shall be using later.

Lemma 1. Let $0<x \leq 1$ be a real number. Then $\sum_{n=0}^{\infty} 2^{n} x^{2^{n}} \leq \frac{x(1+x)}{(1-x)}$

Proof. Note that

$$
2^{n} x^{2^{n}} \leq 2 \sum_{2^{n-1}<j \leq 2^{n}} x^{j}
$$

Summing over $n=1$ to $\infty$,

$$
\sum_{n=1}^{\infty} 2^{n} x^{2^{n}} \leq 2 \sum_{j=2}^{\infty} x^{j}=\frac{2 x^{2}}{1-x} .
$$

Adding $x$ (corresponding to $n=0$ ) on both sides, we get the result.

Note that $\psi:(0, \infty) \rightarrow \mathbb{R}$ be a continuous function, which is positive and increasing. It can be shown that if the complement of $\mathscr{A}$ is finite then

$$
\psi(Y)^{2}=\left(2 Y+O\left(\frac{1}{Y}\right)\right) \psi\left(\frac{Y}{2}\right) .
$$

and conversely.

We aim to prove that, even if $\Psi(Y)$ satisfies a hypothesis, slightly weaker than (4), then some colclusion can be arrived about $\Psi(Y)$.

Let $f: \mathbb{R} \mapsto[0, \infty)$ be an function. For any real number $y$, and integer $\alpha \geq 0$, define $F(y, \alpha)$ by recurrence, as follows:

$$
F(y ; 0)=0 ;
$$

and

$$
F(y, \alpha+1)=\frac{f\left(y 2^{\alpha+1}\right)+F(y, \alpha)}{2} .
$$

In other words,

$$
F(y ; \alpha)=\frac{f\left(2^{\alpha} y\right)}{2}+\frac{f\left(2^{\alpha-1} y\right)}{4}+\frac{f\left(2^{\alpha-2} y\right)}{8}+\ldots . .+\frac{f(2 y)}{2^{\alpha}}
$$

Lemma 2. If $(\psi(Y))^{2} \geq 2 Y \exp (-f(Y)) \psi\left(\frac{Y}{2}\right)$ for all $Y \geq N_{0}$, then

$$
\psi\left(y 2^{\alpha}\right) \geq y 2^{\alpha} \exp (-F(y ; \alpha))\left(\frac{\psi(y)}{y}\right)^{\frac{1}{2^{\alpha}}}
$$

for all integers $\alpha \geq 0$. and a fixed real number $y \geq N_{0}$ 
Proof. For $\alpha=0$, both sides are equal; For the general case by induction,

$$
\begin{aligned}
\left(\psi\left(y 2^{\alpha+1}\right)\right)^{2} & \geq 2 y 2^{\alpha+1} \exp \left(-f\left(y 2^{\alpha+1}\right)\right) \psi\left(y 2^{\alpha}\right) \\
& =y^{2} 2^{2 \alpha+2} \exp \left(-f\left(y 2^{\alpha+1}\right)-F(y ; \alpha)\right)\left(\frac{\psi(y)}{y}\right)^{\frac{1}{2^{\alpha}}} \\
& =\left(r 2^{\alpha+1} \exp (-F(y, \alpha+1))\left(\frac{\psi(y)}{y}\right)^{\frac{1}{2^{\alpha+1}}}\right)^{2}
\end{aligned}
$$

and hence the result.

We will see the implication of Lemma 1 and Lemma 2 in the proof of Theorem 1 . Before doing so we need one more fact.

Intuitively it makes sense that if $T(Y)(=T([Y]))$ is 'small' then $g(Y)$ should also be 'small. We will show that is the case in the next lemma:

Lemma 3. Let $g(Y)$ and $T(Y)$ are defined as above. Then

$$
g(Y)<T(Y)+10
$$

for all $Y \geq 40$. Further if $T(N) \leq \frac{1}{36} \mathscr{A}(N)$ for all integers $N \geq N_{0}$ then there exists $N_{2} \geq N_{0}$ such that

$$
g(Y) \leq \psi\left(\frac{Y}{2}\right)
$$

for all $Y \geq N_{2}$

Proof. First take $N=[Y]$. Then we have:

$$
\begin{aligned}
\left(1-e^{-\frac{2}{N}}\right) \sum_{k=1}^{\infty} S_{k} e^{-\frac{2 k}{N}}= & \sum_{k=1}^{\infty}\left(R_{2}(2 k)-R_{2}(2 k+1)\right) e^{-\frac{2 k}{N}} \\
= & \sum_{k=1}^{m(N)}\left(R_{2}(2 k)-R_{2}(2 k+1)\right) e^{-\frac{2 k}{N}} \\
& +\sum_{k=m(N)}^{\infty}\left(R_{2}(2 k)-R_{2}(2 k+1)\right) e^{-\frac{2 k}{N}} \\
= & \Sigma_{1}+\Sigma_{2} \text { Say; }
\end{aligned}
$$

Now

$$
\begin{aligned}
\Sigma_{1} & =\sum_{k=1}^{m(N)}\left(R_{2}(2 k)-R_{2}(2 k+1)\right) e^{-\frac{2 k}{N}} \\
& =\left(1-e^{-\frac{2}{N}}\right) \sum_{k=1}^{m(N)-1} S_{k} e^{-\frac{2 k}{N}}+S_{m(N)} e^{-\frac{m(N)}{N}} ; \\
& \leq \max _{k \leq m(N)} S_{k}+2 ; \\
& =T(N)+2 .
\end{aligned}
$$

Also

$$
\Sigma_{2}=\sum_{k=m(N)+1}^{\infty}\left(R_{2}(2 k)-R_{2}(2 k+1)\right) e^{-\frac{2 k}{N}}
$$

Using $\left|R_{2}(2 k)-R_{2}(2 k+1)\right|<k \quad$ and $\quad m(N)=N(\log N+\log \log N)$, and also considering the fact that $h_{1}(x)=$ $x e^{-\frac{2 x}{N}}$ is a decreasing function of $x \geq N$ we get,

$$
\begin{aligned}
\Sigma_{2} & \leq \int_{m(N)}^{\infty} x e^{-\frac{2 x}{N}} d x \\
& <\frac{3}{4 \log N} \quad \text { if } N>80
\end{aligned}
$$


So

$$
\begin{aligned}
g(N) & =1+4\left(\Sigma_{1}+\Sigma_{2}\right) ; \\
& <1+4\left(T(N)+\frac{3}{4 \log N}+2\right) \quad \text { for } N \geq N_{0} ; \\
& =4 T(N)+9+\frac{3}{\log N} .
\end{aligned}
$$

Which proves the first part of the lemma since $g$ is an increasing function.

To prove the second part note that

$$
g(Y) \leq \frac{1}{9} \mathscr{A}(Y)+10
$$

for $N \geq 100$. Then the fact that

$$
\Psi\left(\frac{Y}{2}\right)>\sum_{\substack{a \in \mathscr{A} \\ a \leq Y}} e^{-\frac{a}{Y}}>e^{-2} \sum_{\substack{a \in \mathscr{A} \\ a \leq Y}} 1=\frac{1}{e^{2}} \mathscr{A}(Y),
$$

proves the result.

\section{Proof of Theorem 1;}

We observe, by comparing the coefficients of $\alpha^{n}$ from both sides, that

$$
f\left(\alpha^{2}\right)=\frac{1-\alpha}{2 \alpha}(f(\alpha))^{2}+2 \sum_{k=1}^{\infty}\left(R_{2}(2 k)-R_{2}(2 k+1)\right) \alpha^{2 k}-\frac{(1+\alpha)}{2 \alpha} f(-\alpha)^{2} .
$$

Since $\alpha>0$ this gives,

$$
f\left(\alpha^{2}\right) \leq \frac{1-\alpha}{2 \alpha} f(\alpha)^{2}+2 \sum_{k=1}^{\infty}\left(R_{2}(2 k)-R_{2}(2 k+1)\right) \alpha^{2 k}
$$

Now considering the right hand side of the summation, we get:

$$
\begin{aligned}
\sum_{k=1}^{\infty}\left(R_{2}(2 k)-R_{2}(2 k+1)\right) \alpha^{2 k} & =\sum_{k=1}^{\infty}\left(S_{k}-S_{k-1}\right) \alpha^{2 k} \\
& =\sum_{k=1}^{\infty} S_{k}\left(\alpha^{2 k}-\alpha^{2 k+1}\right) \\
& =\left(1-\alpha^{2}\right) \sum_{k=1}^{\infty} S_{k} \alpha^{2 k}
\end{aligned}
$$

Thus we get, from (5),

$$
f\left(\alpha^{2}\right) \leq \frac{1-\alpha}{2 \alpha} f(\alpha)^{2}+2\left(1-\alpha^{2}\right) \sum_{k=1}^{\infty} S_{k} \alpha^{2 k}
$$

Now putting $\alpha=e^{\frac{-1}{Y}}$, we get

$$
\psi\left(\frac{Y}{2}\right) \leq \frac{1}{2}\left(\frac{1}{Y}+\frac{1}{Y^{2}}\right)(\psi(Y))^{2}+2\left(1-e^{-\frac{2}{Y}}\right) \sum_{k=1}^{\infty} S_{k} e^{-\frac{2 k}{Y}} .
$$

Since $\psi(Y) \leq Y$, this gives

$$
2 Y \psi\left(\frac{Y}{2}\right) \leq(\psi(Y))^{2}+Y g(Y)
$$

Thus

$$
(\psi(Y))^{2} \geq 2 Y \psi\left(\frac{Y}{2}\right)-Y g(Y)
$$

Now using the second part of lemma 3 , we get

$$
\psi(Y)^{2} \geq Y \psi\left(\frac{Y}{2}\right) \quad \forall Y \geq N_{0}
$$

Thus, $\psi$ satisfies lemma2 with $f(Y)=\log 2$. Thus

$$
F(y ; \alpha)=(\log 2)\left(1-\frac{1}{2^{\alpha+1}}\right)
$$


Thus,

$$
\psi\left(y_{0} 2^{\alpha}\right) \geq \frac{y_{0}}{2} 2^{\frac{1}{2^{\alpha+1}}}\left(\frac{\psi\left(y_{0}\right)}{y_{0}}\right)^{\frac{1}{2^{\alpha}}} \text { for } N_{0} \leq y_{0}<2 N_{0}
$$

This gives, since $\psi\left(N_{0}\right) \geq 1$, and if $\alpha$ is sufficently large, say $\alpha \geq \alpha_{0}$,

$$
\psi\left(y_{0} 2^{\alpha}\right) \geq(0.49) y_{0} 2^{\alpha}
$$

for $y_{0} \geq N_{0}$. Thus choosing $\alpha$ suitably and defining $y_{0}=\frac{Y}{2^{\alpha}}$ so that $N_{0} \leq y_{0}<2 N_{0}$, we get

$$
\psi(Y) \geq(0.49) Y \quad \text { for all } Y \geq N_{1}\left(=N_{0} 2^{\alpha_{0}}\right) .
$$

Thus, from (8)

$$
\psi(Y)^{2} \geq 2 Y \psi\left(\frac{Y}{2}\right)\left(1-\frac{g(Y)}{0.49 Y}\right) .
$$

for all $Y \geq N_{1}$. Since $\frac{g(Y)}{Y}<\frac{1}{9}$, lemma2 is satisfied with $f(Y)=2.3 \frac{g(Y)}{Y}$

Then

$$
F(y ; \alpha)=\frac{2.3}{2^{\alpha+1} y}\left(g\left(2^{\alpha} y\right)+g\left(2^{\alpha-1} y\right)+\ldots . .+g(2 y)\right)
$$

This gives

$$
\psi\left(y_{1} 2^{\alpha}\right) \geq y_{1} 2^{\alpha} \exp \left(-F\left(y_{1} ; \alpha\right)\right)\left(\frac{\psi\left(y_{1}\right)}{y_{1}}\right)^{\frac{1}{2^{\alpha}}}
$$

for $N_{1} \leq y_{1}<2 N_{1}$.

Note that in this case

$$
\begin{aligned}
g\left(2^{\alpha} y_{1}\right)+g\left(2^{\alpha-1} y_{1}\right)+\ldots+g\left(2 y_{1}\right) & =\alpha+\frac{8}{2^{\alpha} y_{1}} \sum_{k=1}^{\infty} S_{k}^{+} \sum_{n=0}^{\alpha} 2^{n} e^{-\frac{2^{n+1} y_{k}}{y_{1} 2^{\alpha}}} \\
& \leq \alpha+\frac{8}{2^{\alpha} y_{1}} \sum_{k=1}^{\infty} S_{k}^{+} \frac{\left.e^{-\frac{2 k}{2^{\alpha} y_{1}}\left(1+e^{-\frac{2 k}{2^{\alpha} y_{1}}}\right.}\right)}{1-e^{-\frac{2 k}{2^{\alpha} y_{1}}}} ; \quad \text { by Lemma1 } 1 \\
& \leq \alpha+\frac{8}{2^{\alpha} y_{1}} \sum_{k=1}^{\infty} S_{k}^{+} \frac{2^{\alpha} y_{1}}{k}
\end{aligned}
$$

That implies for large enough $\alpha$ and fixed $y_{1}$ with $N_{1} \leq y_{1}<2 N_{1}$,

$$
\frac{\psi\left(y_{1} 2^{\alpha}\right)}{y_{1} 2^{\alpha}} \geq \exp \left(-F\left(y_{1} ; \alpha\right)+\frac{c}{y_{1} 2^{\alpha}}\right)
$$

for some constant $c$ depending on $\mathscr{A}$. For example, you can choose $c=\inf _{N_{1} \leq y<2 N_{1}} y \log \left(\frac{\psi(y)}{y}\right)$.

Taking logarithm on both sides

$$
F\left(y_{1} ; \alpha\right)-\frac{c}{y_{1} 2^{\alpha}}>\left(1-\frac{\psi\left(y_{1} 2^{\alpha}\right)}{y_{1} 2^{\alpha}}\right),
$$

Or,

$$
\frac{2.3}{2^{\alpha+1} y_{1}}\left(\alpha+8 \sum_{k=1}^{\infty} \frac{S_{k}^{+}}{k}\right)-\frac{c}{y_{1} 2^{\alpha}} \geq \frac{1}{e}\left(\frac{y_{1} 2^{\alpha}-\mathscr{A}\left(y_{1} 2^{\alpha}\right)}{y_{1} 2^{\alpha}}\right) .
$$

As before choosing $\alpha$ suitably, so that $N_{1} \leq y_{1}\left(=\frac{N}{2^{\alpha}}\right)<2 N_{1}$, it implies that

$$
\sum_{k=1}^{\infty} \frac{S_{k}^{+}}{k}>\frac{1}{10 e}(N-\mathscr{A}(N))-\frac{1}{7} \log N-c_{1}
$$

for large enough $N$ and fixed constant $c_{1}$ depending on $\mathscr{A}$. Which proves Theorem 1 , 


\section{REMARK 2}

Remark 2. Also we sloved a question raised by Sárközy (see [10])[Problem 5, Page 337]: Does there exist a set $\mathscr{A} \subset \mathbb{N}$ such that $\mathscr{A}$ is an infinite set and $R_{1}(n+1) \geq R_{1}(n)$ holds on a sequence of integers $n$ whose density is 1 ? Here we show that the answer to this question is positive by giving a simple example.

Let $\mathscr{B}$ be an infinite Sidon set of even integers and $\mathscr{A}=\mathbb{N} \backslash \mathscr{B}$;

Put

$$
Y=(\mathscr{B}+\mathscr{B}) \cup \mathscr{B} \text { and } X=\mathbb{N} \backslash Y ;
$$

Then,

$$
R_{1}(n+1) \geq R_{1}(n) \quad \text { for all } n \in X
$$

To see this, let

$$
\begin{aligned}
& f(z)=\sum_{a \in \mathscr{A}} z^{a} \quad \text { and } g(z)=\sum_{b \in \mathscr{B}} z^{b}, \\
&\text { Then } \left.\sum_{n=1}^{\infty}\left(R_{(} n\right)-R_{1}(n-1)\right) z^{n}=(1-z) f(z)^{2} \\
&=(1-z)\left(\frac{z}{(1-z)}-g(z)\right)^{2} \\
&=\frac{z^{2}}{(1-z)}+(1-z)(g(z))^{2}-2 z g(z)
\end{aligned}
$$

let

$$
r_{1}(n)=\sum_{\substack{b_{i}+b_{j}=n \\ b_{i} \in \mathscr{B}, b_{j} \in \mathscr{B}}} 1,
$$

So $R_{1}(n+1) \geq R_{1}(n)$ iff coefficient of $z^{n+1}$ in $(1-z)(f(z))^{2}$ is non negetive.

$$
\begin{aligned}
\text { Now coefficient of } z^{2 k} \text { is } & =1+r_{1}(2 k)-r_{1}(2 k-1)-2 \chi_{\mathscr{B}}(2 k-1) \\
\text { and coefficient of } z^{2 k+1} \text { is } & =1+r_{1}(2 k+1)-r_{1}(2 k)-2 \chi_{\mathscr{B}}(2 k)
\end{aligned}
$$

Then it is clear from the above choice of $X$ and $\mathscr{A}$ that, $R_{1}(n+1) \geq R_{1}(n)$ for all $n$ in $X$.

For example we can take $\mathscr{B}=\left\{2,4,8,16,32, \ldots ., 2^{m}, \ldots.\right\}$. Then $\mathscr{B}$ is infinite and $X$ is of density 1 .

\section{REFERENCES}

[1] M. Ajtai, J. Komlós and E. Szemerédi, 'A dense infinite Sidon sequence', European J. Combin. 2 (1981), 1-11.

[2] R. Balasubramanian, 'A note on a result of Erd"os, S'ark"ozy and S'os,' Acta Arith. 49 (1987), 45-53.

[3] Yong-Gao Chen, András Sárközy, Vera T. Sós and Min Tang, 'On The Monotonicity Properties of Additive Representations Functions', Bull. Austral. Math. Soc., 72 (2005), 129-138.

[4] Min Tang and Yong-Gao Chen, 'On Additive Properties of General Sequences.' Bull. Austral. Math. Soc., 71, (2005) 479-485

[5] P. Erdős and A. Sárközy, 'Problems and results on additive properties of general sequences,' P, Pacific J. Math. 118 (1985), $347-357$.

[6] P. Erdős and A. Sárközy, 'Problems and results on additive properties of general sequences, II', Ada Math. Hungar. 48 (1986), $201-211$.

[7] P. Erdôs, A. Sárközy and V.T. Sós, 'Problems and results on additive properties of general sequences, III', Studia Sci. Math. Hungar. 22 (1987), 53-63.

[8] P. Erdős, A. Sárközy and V.T. Sós, 'Problems and results on additive properties of general sequences, IV,', in Number Theory, Proceedings, Ootacamund, India, 1984, Lecture Notes in Mathematics 1122 (Springer-Verlag, Berlin, 1985), pp. 85-104.

[9] P. Erdős, A. Sárközy and V.T. Sós, 'Problems and results on additive properties of general sequences, V', Monatsh. Math. 102 (1986), 183-197.

[10] A. Sárközy, 'On the Number of Additive Representations of Integers.' Bolyai Society Mathematical Studies, 15, (2006) 329-339. 


\title{
ON ADDITIVE REPRESENTATION FUNCTIONS
}

\author{
R. BALASUBRAMANIAN AND SUMIT GIRI
}

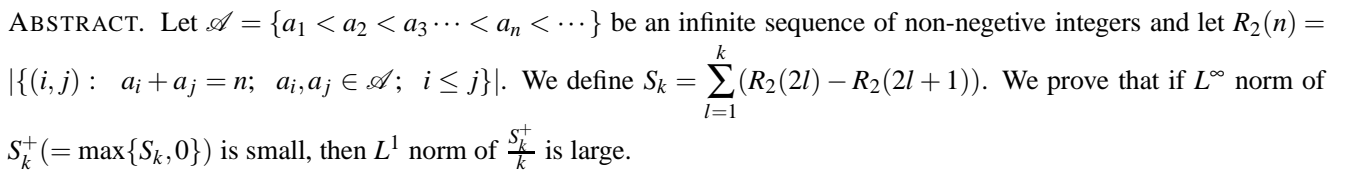

\section{INTRODUCTION}

Let $\mathscr{A}=\left\{a_{1}, a_{2}, \cdots\right\}\left(0 \leq a_{1}<a_{2}<\cdots\right)$ be an infinite sequence of non-negative integers. For $n \in \mathbb{N}_{0}$, Define

$$
\begin{aligned}
& R_{1}(n)=R_{1}(\mathscr{A}, n)=\sum_{a_{i}+a_{j}=n} 1 \\
& R_{2}(n)=R_{2}(\mathscr{A}, n)=\sum_{\substack{a_{i}+a_{j}=n \\
i \leq j}} 1 .
\end{aligned}
$$

Now, it is easy to check that if $\mathscr{A}$ is a full set or a complement of a finite set inside the set of natural numbers, then $R_{1}$ and $R_{2}$ are monotonically increasing. Here we are interested in inverse problems, i.e., how the monotonicity of the representation functions affects the cardinality of the set $\mathscr{A}$.

The question of characterisation of the set $\mathscr{A}$, under the condition that either $R_{1}(n)$ or $R_{2}(n)$ is monotonic, was raised by Erdős, Sárközy and Sós [8]. Also see [9] and [2].

In [8], Erdős, Sárközy and Sós proved that $R_{1}(n)$ can be monotonically increasing from a certain point, only in a trivial way. See [8] and [2] for the following theorem.

Theorem A. If $R_{1}(n+1) \geq R_{1}(n)$ for all large $n$, then $\mathbb{N} \backslash \mathscr{A}$ is a finite set.

While analogous conclusion is not true in case of $R_{2}$.

If, we define

$$
\mathscr{A}(N)=|\mathscr{A} \cap[1, N]|,
$$

then the first author [2] proved the following theorem:

Theorem B. If $R_{2}(n+1) \geq R_{2}(n)$ for all large $n$, then $\mathscr{A}(N)=N+O(\log N)$.

In other words, If $R_{2}(n)$ is monotonic, then the complement set of $\mathscr{A}$ is almost of order $O(\log N)$.

In the first part of this paper we shall focus on the function $R_{2}$ and quantities related to monotonicity of it. Also in Section 6, we shall make a remark concerning a question raised by Sárközy [10], related to monotonicity of $R_{1}$.

In [9] Erdős, Sárközy and Sós proved

Theorem C. If

$$
\lim _{n \rightarrow+\infty} \frac{n-\mathscr{A}(n)}{\log n}=+\infty
$$

then we have,

$$
\limsup _{N \rightarrow+\infty} \sum_{k=1}^{N}\left(R_{2}(2 k)-R_{2}(2 k+1)\right)=+\infty .
$$

The assumption (4) in the above theorem can not be relaxed. In fact Erdôs, Sárközy and Sós [9] constructed a sequence $\hat{\mathscr{A}}$ where $(n-\hat{\mathscr{A}}(n))>c \log n$ (for large $n$ and fixed constatnt $c$ ) and

$$
\limsup _{N \rightarrow+\infty} \sum_{k=1}^{N}\left(R_{2}(2 k)-R_{2}(2 k+1)\right)<+\infty .
$$


In [4], Tang and Chen gave a quantative version of Theorem C] Before we state their theorem, let us define a few notations.

$$
\begin{aligned}
S_{n} & =\sum_{k \leq n}\left(R_{2}(2 k)-R_{2}(2 k+1)\right), \\
m(N) & =N(\log N+\log \log N) .
\end{aligned}
$$

Also $L^{\infty}$ norm of $S_{n}$, denoted by $T(N)$, is defined as follow:

$$
T(N)=\max _{n \leq m(N)} S_{n}=\max _{n \leq m(N)} \sum_{k \leq n}\left(R_{2}(2 k)-R_{2}(2 k+1)\right) .
$$

In [4] the authors proved that, when the ratio $\frac{T(N)}{\mathscr{A}(N)}$ is bounded above by a small enough fixed constatnt, then $T(N)$ and $\frac{N-A(N)}{\log N}$ satisfies a simple inequality. More precisely,

Theorem D. If $T(N)$ be defined as in (6) and

$$
T(N)<\frac{1}{36} \mathscr{A}(N)
$$

for all large enough $N$, then there exists a $C>0$, depending only on $\mathscr{A}$, such that

$$
T(N)>\frac{1}{80 e} \frac{N-\mathscr{A}(N)}{\log N}-\frac{11}{4}-\frac{C}{8}
$$

for all large enough $N$.

It is easy to see that, under the condition (7), Theorem Dimplies Theorem C Now, set

$$
S_{n}^{+}=\max \left\{S_{n}, 0\right\}
$$

and

$$
T^{+}(N)=\max _{n \leq m(N)}\left\{S_{n}^{+}\right\} .
$$

Note: $T(N)$ and $T^{+}(N)$ are same unless all the elements of the set $\left\{S_{n}: n \leq m(N)\right\}$ are negative.

In this paper, we again assume that $\frac{T(N)}{\mathscr{A}(N)}$ is bounded above and prove an improved version of $(8)$ where we replace $L^{\infty}$ norm of $S(n)$ by $L^{1}$ norm of $\frac{S^{+}(n)}{n}$. More precisely, we prove the following theorem:

Theorem 1. Let $\mathscr{A}$ be an infinite sequence of positive integers and there exists $N_{0}$ such that $T(N)<\frac{1}{36} \mathscr{A}(N)$ for all $N \geq N_{0}$. Then there exists a constatnt $c_{1}>0$, depending on $\mathscr{A}$, such that

$$
\sum_{n=1}^{m(N)} \frac{S_{n}^{+}}{n}>\frac{1}{10 e}(N-\mathscr{A}(N))-\frac{1}{4} \log N-c_{1} .
$$

for all large enough $N$.

Corollary 1. If (7) in Theorem $\mathrm{D}$ holds, then for any $\varepsilon>0$,

$$
T^{+}(N)>\frac{1}{10 e+\varepsilon} \frac{N-\mathscr{A}(N)}{\log N}-\frac{1}{4} .
$$

for any large enough $N$.

So, if at least one of $S(n)$ is non-negetive, then $T^{+}(N)$ indeed equals $T(N)$. In that case, Corollary $\square$ gives Theorem $D$ with a better constant. Corollary 1 also implies the following:

Corollary 2. If $\limsup _{N \rightarrow+\infty} \frac{N-\mathscr{A}(N)}{\log N}=+\infty$, then

$$
\limsup _{n \rightarrow+\infty}\left\{S_{n}\right\}=+\infty \text {. }
$$




\section{Generating Functions:}

It is more natural to consider the problem in terms of generating function.

Set

$$
f(z)=\sum_{a \in \mathscr{A}} z^{a}, \quad|z|<1 .
$$

Then,

$$
f(z)^{2}=\sum_{n=1}^{+\infty} R(n) z^{n} .
$$

For any positive real number $Y$, define

$$
\psi(Y)=f\left(e^{-\frac{1}{Y}}\right)=\sum_{a \in \mathscr{A}} e^{-\frac{a}{Y}},
$$

and

$$
g(Y)=1+4\left(1-e^{-\frac{2}{Y}}\right) \sum_{k=1}^{+\infty} S_{k} e^{-\frac{2 k}{Y}} .
$$

Theorem 2. Let $g(Y)$ and $\psi(Y)$ be defined as above. Also assume

$$
g(Y) \leq \min \left\{\psi\left(\frac{Y}{2}\right), \frac{1}{9} Y\right\}
$$

for all sufficiently large positive real number $Y$. Then

$$
\psi(Y) \geq Y \exp \left(-\left(\frac{2.3}{2 Y}\left(\log _{2} Y+\frac{16}{Y} \sum_{k=1}^{\infty} S_{k}^{+} \frac{e^{-\frac{2 k}{Y}}}{1-e^{-\frac{2 k}{Y}}}\right)\right)-\frac{c}{Y}\right)
$$

for some positive constatnt c depending only on first few elements of $\mathscr{A}$.

In Section 3, we will give a proof of Theorem 2 . In Section 4, we will show how Theorem $\square$ follows from Theorem 2

\section{Notations and prelimanary lemmas:}

Consider a function $h: \mathbb{R} \mapsto[0,+\infty)$. For any real number $Y$ and integer $\alpha \geq 0$ define $H(Y ; \alpha)$ by recurrence, as follows:

$$
\begin{aligned}
H(Y ; 0) & =0 \\
H(Y ; \alpha) & =\frac{h(Y)}{2}+\frac{h\left(\frac{Y}{2}\right)}{4}+\frac{h\left(\frac{Y}{4}\right)}{8}+\cdots+\frac{h\left(\frac{Y}{2^{\alpha-1}}\right)}{2^{\alpha}} \\
& =\sum_{j=0}^{\alpha-1} \frac{1}{2^{j+1}} h\left(\frac{Y}{2^{j}}\right) \quad \text { for integer } \alpha \geq 1 .
\end{aligned}
$$

Also

$$
H(Y)=\sum_{j=0}^{\infty} \frac{1}{2^{j+1}} h\left(\frac{Y}{2^{j}}\right) .
$$

Lemma 1. If $h(Y)$ and $H(Y ; \alpha)$ is defined as above and

$$
(\psi(Y))^{2} \geq 2 Y \exp (-h(Y)) \psi\left(\frac{Y}{2}\right)
$$

for all real number $Y \geq \tilde{N}_{0}$, then for every integer $\alpha \geq 0$,

$$
\psi(Y) \geq Y \exp (-H(Y ; \alpha))\left(\frac{\psi\left(\frac{Y}{2^{\alpha}}\right) 2^{\alpha}}{Y}\right)^{\frac{1}{2^{\alpha}}}
$$

for any real number $Y \geq 2^{\alpha} \tilde{N}_{0}$. 
Proof. For $\alpha=0$, both sides are equal.

For the general case, suppose it is true for $\alpha=\alpha_{0}$. Then

$$
\begin{aligned}
(\psi(Y))^{2} & \geq 2 Y \exp (-h(Y)) \psi\left(\frac{Y}{2}\right) \\
& =Y^{2} \exp \left(-h(Y)-H\left(\frac{Y}{2} ; \alpha_{0}\right)\right)\left(\frac{\psi\left(\frac{Y}{2^{\alpha_{0}+1}}\right) 2^{\alpha_{0}+1}}{Y}\right)^{\frac{1}{2^{\alpha_{0}}}} \text { for } Y \geq 2 N_{1} \\
& =\left(Y \exp (-H(Y, \alpha+1))\left(\frac{\psi\left(\frac{Y}{2^{\alpha_{0}+1}}\right) 2^{\alpha_{0}+1}}{Y}\right)^{\frac{1}{2^{\alpha_{0}+1}}}\right)^{2}
\end{aligned}
$$

and hence the result.

Lemma 2. There exist a $c>0$ such that, if $Y$ is larghe enough, then we have

$$
\left(\frac{\psi\left(\frac{Y}{2^{\alpha}}\right) 2^{\alpha}}{Y}\right)^{\frac{1}{2^{\alpha}}} \geq \exp \left(-\frac{c}{Y}\right)
$$

for some $\alpha \leq \log _{2} Y$.

Proof. Now fix an interval $[a, 2 a]$ so that $\psi(a) \geq 1$.

Then choose $\alpha$ suitably so that $\frac{Y}{2^{\alpha}} \in[a, 2 a]$. In that case, we have

$$
\left(\frac{\psi\left(\frac{Y}{2^{\alpha}}\right) 2^{\alpha}}{Y}\right)^{\frac{Y}{2^{\alpha}}} \geq\left(\frac{1}{2 a}\right)^{2 a}
$$

This proves the lemma.

Lemma 3. Let $0<x<1$ be a real number. Then $\sum_{n=0}^{+\infty} 2^{n} x^{2^{n}} \leq \frac{2 x}{(1-x)}$.

Proof. Note that

$$
2^{n} x^{2^{n}} \leq 2 \sum_{2^{n-1}<j \leq 2^{n}} x^{j}
$$

Summing over $n=1$ to $+\infty$,

$$
\sum_{n=1}^{+\infty} 2^{n} x^{2^{n}} \leq 2 \sum_{j=2}^{+\infty} x^{j}=\frac{2 x^{2}}{1-x} .
$$

Adding $x$ (corresponding to $n=0$ ) on both sides, we get the result.

Lemma 4. In the notaion of Lemma1 let $h(Y)=d \frac{g(Y)}{Y}$ for some fixed positive constant $d$, to be choosen later. Then

$$
H(Y, \alpha) \leq \frac{d}{2 Y}\left(\alpha+\frac{8}{Y} \sum_{k=1}^{\infty} S_{k}^{+} \frac{e^{-\frac{2^{k+1}}{Y}}}{1-e^{-\frac{2^{k+1}}{Y}}}\right)
$$

Proof. set $x=e^{-\frac{2}{Y}}$.

$$
\begin{aligned}
g(Y) & =1+4\left(1-e^{-\frac{2}{Y}}\right) \sum_{k=1}^{\infty} S_{k}^{+} x^{k} . \\
& \leq 1+\frac{8}{Y} \sum_{k=1}^{\infty} S_{k}^{+} x^{k} .
\end{aligned}
$$


Then

$$
\begin{aligned}
H(Y ; \alpha) & =\sum_{j=0}^{\alpha-1} \frac{1}{2^{j+1}} h\left(\frac{Y}{2^{j}}\right) \\
& \leq \sum_{j=0}^{\alpha-1} \frac{d}{2 Y}\left(1+\frac{8}{Y} \sum_{k=1}^{\infty} S_{k}^{+} 2^{j} x^{2^{j} k}\right) \\
& \leq \frac{d}{2 Y}\left(\alpha+\frac{8}{Y} \sum_{k=1}^{\infty} S_{k}^{+} \sum_{j=0}^{\infty} 2^{j} x^{2^{j} k}\right) \\
& =\frac{d}{2 Y}\left(\alpha+\frac{8}{Y} \sum_{k=1}^{\infty} S_{k}^{+} \frac{2 x^{k}}{1-x^{k}}\right) .
\end{aligned}
$$

\section{Proof of Theorem 2:}

It is easy to verify the following inequality by comparing the coefficients of $z^{n}$ from both sides.

$$
f\left(z^{2}\right)=\frac{1-z}{2 z}(f(z))^{2}+2 \sum_{k=1}^{+\infty}\left(R_{2}(2 k)-R_{2}(2 k+1)\right) z^{2 k}-\frac{(1+z)}{2 z} f(-z)^{2} .
$$

If $z>0$, this gives,

$$
f\left(z^{2}\right) \leq \frac{1-z}{2 z} f(z)^{2}+2 \sum_{k=1}^{+\infty}\left(R_{2}(2 k)-R_{2}(2 k+1)\right) z^{2 k} .
$$

Now, considering the right hand side of the summation, we get

$$
\begin{aligned}
\sum_{k=1}^{+\infty}\left(R_{2}(2 k)-R_{2}(2 k+1)\right) z^{2 k} & =\sum_{k=1}^{+\infty}\left(S_{k}-S_{k-1}\right) z^{2 k} \\
& =\sum_{k=1}^{+\infty} S_{k}\left(z^{2 k}-z^{2 k+2}\right)-S_{0} z^{2} \\
& \leq\left(1-z^{2}\right) \sum_{k=1}^{+\infty} S_{k} z^{2 k}
\end{aligned}
$$

Thus, from (20) we get

$$
f\left(z^{2}\right) \leq \frac{1-z}{2 z} f(z)^{2}+2\left(1-z^{2}\right) \sum_{k=1}^{+\infty} S_{k} z^{2 k}
$$

Now putting $z=e^{\frac{-1}{Y}}$, we get

$$
\psi\left(\frac{Y}{2}\right) \leq \frac{1}{2}\left(\frac{1}{Y}+\frac{1}{Y^{2}}\right)(\psi(Y))^{2}+2\left(1-e^{-\frac{2}{Y}}\right) \sum_{k=1}^{+\infty} S_{k} e^{-\frac{2 k}{Y}} .
$$

Since $\psi(Y) \leq Y$, this gives

$$
2 Y \psi\left(\frac{Y}{2}\right) \leq(\psi(Y))^{2}+Y g(Y)
$$

Thus,

$$
(\psi(Y))^{2} \geq 2 Y \psi\left(\frac{Y}{2}\right)-Y g(Y) .
$$

Lemma 5. If $g(Y) \leq \psi\left(\frac{Y}{2}\right)$, then for all large enough real number $Y$

$$
\psi(Y) \geq 0.49 Y \text {. }
$$

Proof. Since $g(Y) \leq \psi\left(\frac{Y}{2}\right)$, using (21) we get

$$
(\psi(Y))^{2} \geq Y \psi\left(\frac{Y}{2}\right)
$$


Then (17) in Lemma $\square$ is holds with $h(Y)=\log 2$.

In that case

$$
H(Y)=\sum_{0 \leq j \leq \infty} \frac{1}{2^{j+1}} h\left(\frac{Y}{2^{j}}\right)=\log 2 .
$$

This gives, by Lemma $\square$ and Lemma 2 ,

$$
\psi(Y) \geq(0.49) Y
$$

if $Y$ is large enough.

Thus, combining (21) and Lemma 5 we get

$$
\psi(Y)^{2} \geq 2 Y \psi\left(\frac{Y}{2}\right)\left(1-\frac{g(Y)}{0.49 Y}\right)
$$

for sufficiently large $Y$.

Since $\frac{g(Y)}{Y}<\frac{1}{9}$, equation (17) in Lemma $\square$ is satisfied with $h(Y)=2.3 \frac{g(Y)}{Y}$.

Hence Lemma 4 and Lemma $\square$ together give the following:

$$
\psi(Y) \geq Y \exp \left(-\frac{2.3}{2 Y}\left(\alpha+\frac{16}{Y} \sum_{k=1}^{\infty} S_{k}^{+}\left(\frac{e^{-\frac{2 k}{N}}}{1-e^{-\frac{2 k}{N}}}\right)\right)\right)\left(\frac{\psi\left(\frac{Y}{2^{\alpha}}\right) 2^{\alpha}}{Y}\right)^{\frac{1}{2^{\alpha}}} .
$$

Hence Theorem 2 follows from (23) and Lemma 2 ,

\section{Proof of Theorem 1}

Lemma 6. Let $g(N)$ and $T(N)$ are defined as in (13) and (6). Then

(a)

$$
g(N)<4 T(N)+40
$$

for all real number $N \geq 40$.

(b) Further, if $T(N) \leq \frac{1}{36} \mathscr{A}(N)$ for all integers $N \geq N_{0}$, then there exists $N_{2} \geq N_{0}$ such that

$$
g(N) \leq \psi\left(\frac{N}{2}\right)
$$

for all real number $N \geq N_{2}$.

Proof. We know $S_{k} \leq T(N)$ for $k \leq m(N)$ and $S_{k} \leq \frac{k^{2}}{2}$ for $k \geq m(N)$.

Now

$$
\begin{aligned}
g(N) & =1+4\left(1-e^{-\frac{2}{N}}\right)\left\{\sum_{k \leq m(N)} S_{k} e^{-\frac{2 k}{N}}+\sum_{k>m(N)} S_{k} e^{-\frac{2 k}{N}}\right\} \\
& =1+4\left(1-e^{-\frac{2}{N}}\right)\left\{\Sigma_{3}+\Sigma_{4}\right\}, \quad \text { say. } \\
& \Sigma_{3} \leq \sum_{k=0}^{\infty} T(N) e^{-\frac{2 k}{N}}=T(N) \frac{1}{1-e^{-\frac{2}{N}}} . \\
& \Sigma_{4} \leq \sum_{k>m(N)} \frac{k^{2}}{2} e^{-\frac{2 k}{N}} \leq \int_{m(N)}^{+\infty} \frac{x^{2}}{2} e^{-\frac{2 x}{N}} d x \leq 10
\end{aligned}
$$

and hence $(a)$. To prove $(b)$ note that

for $N \geq 100$. Then, the fact that

$$
g(N) \leq \frac{1}{9} \mathscr{A}(N)+10
$$

$$
\Psi\left(\frac{N}{2}\right)>\sum_{\substack{a \in \mathscr{A} \\ a \leq N}} e^{-\frac{a}{N}}>e^{-2} \sum_{\substack{a \in \mathscr{A} \\ a \leq N}} 1=\frac{1}{e^{2}} \mathscr{A}(N)
$$

proves the result. 
Lemma 6 shows condition (14) of Theorem 2 are satisfied. Hence

$$
\frac{\psi(N)}{N} \geq \exp \left(-\frac{2.3}{2 N}\left(\log _{2} N+\frac{16}{N} \sum_{k=1}^{\infty} S_{k}^{+} \frac{e^{-\frac{2 k}{N}}}{1-e^{-\frac{2 k}{N}}}\right)-\frac{c}{N}\right)
$$

for some constant $c$ depending on $\mathscr{A}$.

Taking logarithm on both sides,

$$
\frac{2.3}{2 N}\left(\log _{2} N+\frac{16}{N} \sum_{k=1}^{\infty} S_{k}^{+} \frac{e^{-\frac{2 k}{N}}}{1-e^{-\frac{2 k}{N}}}\right)+\frac{c}{N}>\log \left(1-\left(1-\frac{\psi(N)}{N}\right)\right)>\left(1-\frac{\psi(N)}{N}\right) .
$$

Or,

$$
\frac{2.3}{2 N}\left(\log _{2} N+\frac{16}{N} \sum_{k=1}^{m(N)} S_{k}^{+}\left(\frac{e^{-\frac{2 k}{N}}}{1-e^{-\frac{2 k}{N}}}\right)+10\right)+\frac{c}{N}>\left(1-\frac{\psi(N)}{N}\right) .
$$

Now, $\frac{e^{-x}}{1-e^{-x}} \leq \frac{1}{x}$ and hence we can replace $\frac{e^{-\frac{2 k}{N}}}{1-e^{-\frac{2 k}{N}}}$ by $\frac{2 k}{N}$.

$$
\frac{2.3}{2 N}\left(\log _{2} N+8 \sum_{k=1}^{+\infty} \frac{S_{k}^{+}}{k}+10\right)+\frac{c}{N} \geq \frac{1}{e}\left(\frac{N-\mathscr{A}(N)}{N}\right) .
$$

It implies that

$$
\sum_{k=1}^{+\infty} \frac{S_{k}^{+}}{k}>\frac{1}{10 e}(N-\mathscr{A}(N))-\frac{1}{8} \log _{2} N-c_{1}
$$

for large enough $N$ and fixed constant $c_{1}$ depending on $\mathscr{A}$. This proves Theorem $\square$

\section{Monotonicity of $R_{1}(n)$ ON DENSE SET OF INTEGERS}

Remark 1. Also, we solved a question raised by Sárközy (see [10])[Problem 5, Page 337]. His question was the following:

Does there exist an infinite set $\mathscr{A} \subset \mathbb{N}$ such that $\mathbb{N} \backslash \mathscr{A}$ is also an infinite and $R_{1}(n+1) \geq R_{1}(n)$ holds on a sequence of integers $n$ whose density is 1 ?

Here we show that the answer to this question is positive by giving a simple example.

A Sidon set is a set of positive integers such that the sums of any two terms are all different. i.e., $R_{2}(n) \leq 1$ for the corresponding $R_{2}$ function. By [1], it is possible to construct sidon sequence of order $(n \log n)^{\frac{1}{3}}$.

Now, let $\mathscr{B}$ be an infinite Sidon set of even integers and $\mathscr{A}=\mathbb{N} \backslash \mathscr{B}$;

Put

$$
Y=(\mathscr{B}+\mathscr{B}) \cup \mathscr{B} \text { and } X=\mathbb{N} \backslash Y ;
$$

Then,

$$
R_{1}(n+1) \geq R_{1}(n) \quad \text { for all } n \in X
$$

To see this, let

$$
f(z)=\sum_{a \in \mathscr{A}} z^{a} \quad \text { and } g(z)=\sum_{b \in \mathscr{B}} z^{b}
$$

Then,

$$
\begin{aligned}
\sum_{n=1}^{+\infty}\left(R_{1}(n)-R_{1}(n-1)\right) z^{n} & =(1-z) f(z)^{2} \\
& =(1-z)\left(\frac{z}{(1-z)}-g(z)\right)^{2} \\
& =\frac{z^{2}}{(1-z)}+(1-z)(g(z))^{2}-2 z g(z) .
\end{aligned}
$$

Again, let

$$
r_{1}(n)=\sum_{\substack{b_{i}+b_{j}=n \\ b_{i} \in \mathscr{B}, b_{j} \in \mathscr{B} \\ 7}} 1
$$


So, $R_{1}(n+1) \geq R_{1}(n)$ iff coefficient of $z^{n+1}$ in $(1-z)(f(z))^{2}$ is non negetive.

$$
\begin{aligned}
\text { Now coefficient of } z^{2 k} \text { is } & =1+r_{1}(2 k)-r_{1}(2 k-1)-2 \chi_{\mathscr{B}}(2 k-1) \\
\text { and coefficient of } z^{2 k+1} \text { is } & =1+r_{1}(2 k+1)-r_{1}(2 k)-2 \chi_{\mathscr{B}}(2 k)
\end{aligned}
$$

Then, it is clear from the above choice of $X$ and $\mathscr{A}$ that $R_{1}(n+1) \geq R_{1}(n)$ for all $n$ in $X$.

For example, we can take $\mathscr{B}=\left\{2,4,8,16,32, \ldots, 2^{m}, \ldots.\right\}$. Then $\mathscr{B}$ is infinite and $X$ is of density 1 .

\section{REFERENCES}

[1] M. Ajtai, J. Komlós and E. Szemerédi, 'A dense infinite Sidon sequence'. European J. Combin. 2 (1981), 1-11.

[2] R. Balasubramanian, 'A note on a result of Erdős, Sárközy and Sós'. Acta Arith. 49 (1987), 45-53.

[3] Yong-Gao Chen, András Sárközy, Vera T. Sós and Min Tang, 'On The Monotonicity Properties of Additive Representations Functions'. Bull. Austral. Math. Soc., 72 (2005), 129-138.

[4] Min Tang and Yong-Gao Chen, 'On Additive Properties of General Sequences'. Bull. Austral. Math. Soc., 71, (2005) 479-485

[5] P. Erdős and A. Sárközy, 'Problems and results on additive properties of general sequences. I'. Pacific J. Math. 118 (1985), $347-357$.

[6] P. Erdős and A. Sárközy, 'Problems and results on additive properties of general sequences. II'. Acta Math. Hungar. 48 (1986), $201-211$.

[7] P. Erdős, A. Sárközy and V.T. Sós, 'Problems and results on additive properties of general sequences. III'. Studia Sci. Math. Hungar. 22 (1987), 53-63.

[8] P. Erdős, A. Sárközy and V.T. Sós, 'Problems and results on additive properties of general sequences. IV'. Number Theory (Ootacamund, 1984), Lecture Notes in Mathematics 1122. (Springer-Verlag, Berlin, 1985), 85-104.

[9] P. Erdős, Sárközy and V.T. Sós, 'Problems and results on additive properties of general sequences. V'. Monatsh. Math. 102 (1986), 183-197.

[10] A. Sárközy, 'On the Number of Additive Representations of Integers'. Bolyai Society Mathematical Studies, 15, (2006) 329-339. 